\title{
A systematic review of the determinants of seafood consumption
}

\section{Abstract}

Introduction: Little is known about the factors which influence seafood consumption in Irish adults at present. This systematic review will serve the purpose of reviewing published literature examining influences on seafood consumption to provide a basis for a larger project being carried out in UCD - the Seafood Consumption and Risk Exposure Study (SCaRES) - examination of seafood consumption in the Irish population. This project aims to generate food consumption data, on seafood, consumers to improve the characterisation of the risk from exposure to biotoxins and other contaminants from consuming shellfish. This approach will represent a significant step forward in terms of public health policy contribution by being able to characterise the likelihood of illness in a population or population sub-group on an annual basis.

Materials and Methods: Data sources PubMed, Embase, and Web of Science were searched for articles published between January 2008 and January 2018 investigating the characteristics of seafood consumers. Search terms include the following: factors OR influences OR determinants OR indicators AND fish OR seafood OR shellfish OR marine products AND diet OR dietary intake OR intake OR consumption. Articles, in English which investigated factors influencing seafood/fish consumption in adults $(18+)$ were included.

Results: A total of 3964 unique articles were found, after duplicates $(\mathrm{n}=1537)$ were removed. Following this, a further 3531 articles were excluded based on title and abstract. The remaining articles $(n=433)$ were screened based on inclusion criteria. After the initial systematic search, 149 papers met the inclusion criteria. Preliminary analysis to date demonstrated the following; seafood consumers are more likely to have higher educational attainment $(n=43$ article), they are more likely to be of older age ( $n=29$ articles), 21 articles reported that they are less likely to be smokers, whilst $n=17$, found that they are more likely to be physically active, with $n=20$ reporting they are more likely to have a healthier overall diet and $n=14$ reporting that they are more likely to have a higher household income. Results were mixed with respect to BMI.

Discussion: The included studies have found that seafood consumers are more likely to have higher educational attainment, be of older age, have lower tobacco use, be physically active, have a higher fruit and vegetable intake and have a higher household income. Full analysis is ongoing.

\section{Conflict of Interest}

There is no conflict of interest 